\title{
Archéopages
}

Archéopages

Archéologie et société

Hors-série 2 | 2010

Archéologie sans frontières

\section{L'archéologie préventive française, support et modèle pour la formation archéologique en Azerbaïdjan : l'exemple du site de Mentesh-Tépé}

Bertille Lyonnet, Laure Pecqueur, Pascal Raymond et Anaïck Samzun

\section{OpenEdition}

Journals

Édition électronique

URL : https://journals.openedition.org/archeopages/784

DOI : $10.4000 /$ archeopages.784

ISSN : 2269-9872

Éditeur

INRAP - Institut national de recherches archéologiques préventives

Édition imprimée

Date de publication : 1 octobre 2010

Pagination : $72-79$

ISSN : $1622-8545$

Référence électronique

Bertille Lyonnet, Laure Pecqueur, Pascal Raymond et Anaïck Samzun, «L'archéologie préventive française, support et modèle pour la formation archéologique en Azerbaïdjan : l'exemple du site de Mentesh-Tépé », Archéopages [En ligne], Hors-série 2 | 2010, mis en ligne le 01 octobre 2010, consulté le 23 février 2023. URL : http://journals.openedition.org/archeopages/784 ; DOI : https://doi.org/ 10.4000/archeopages.784 

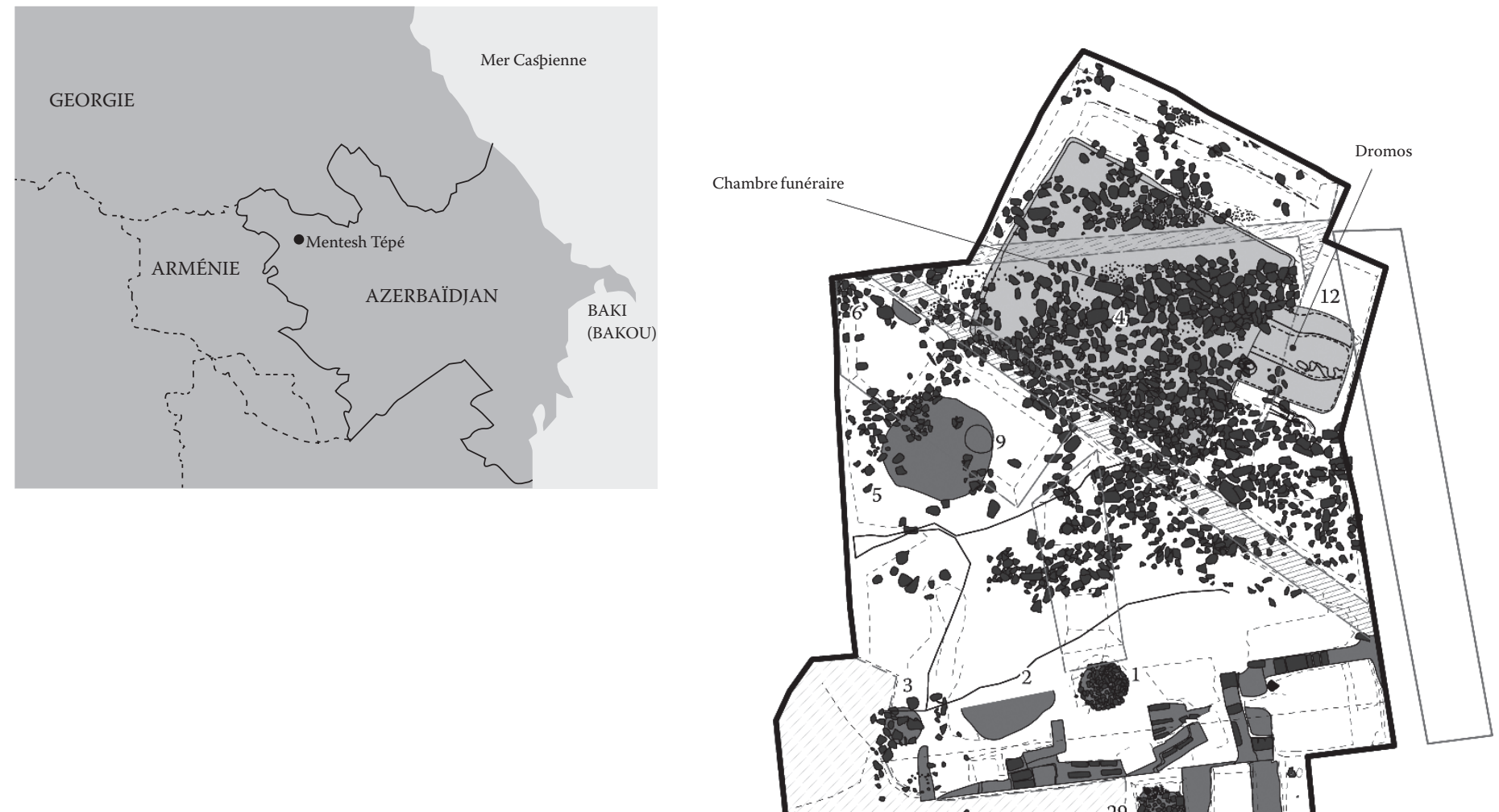

ก

[Fig. 1] Carte de localisation du site de Mentesh-Tépé en Azerbaïdjan.

[Fig.2] Mentesh-Tépé, plan général des vestiges.

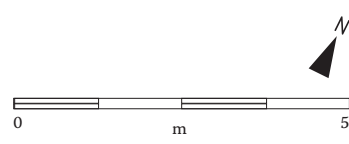

Structures Chalcolithiques.

Structures Kouro-Araxes. Sépulture collective ous kourgan.

$\square$ Sépulture individuelle.

Période indéterminé Perturbation contemporaine.

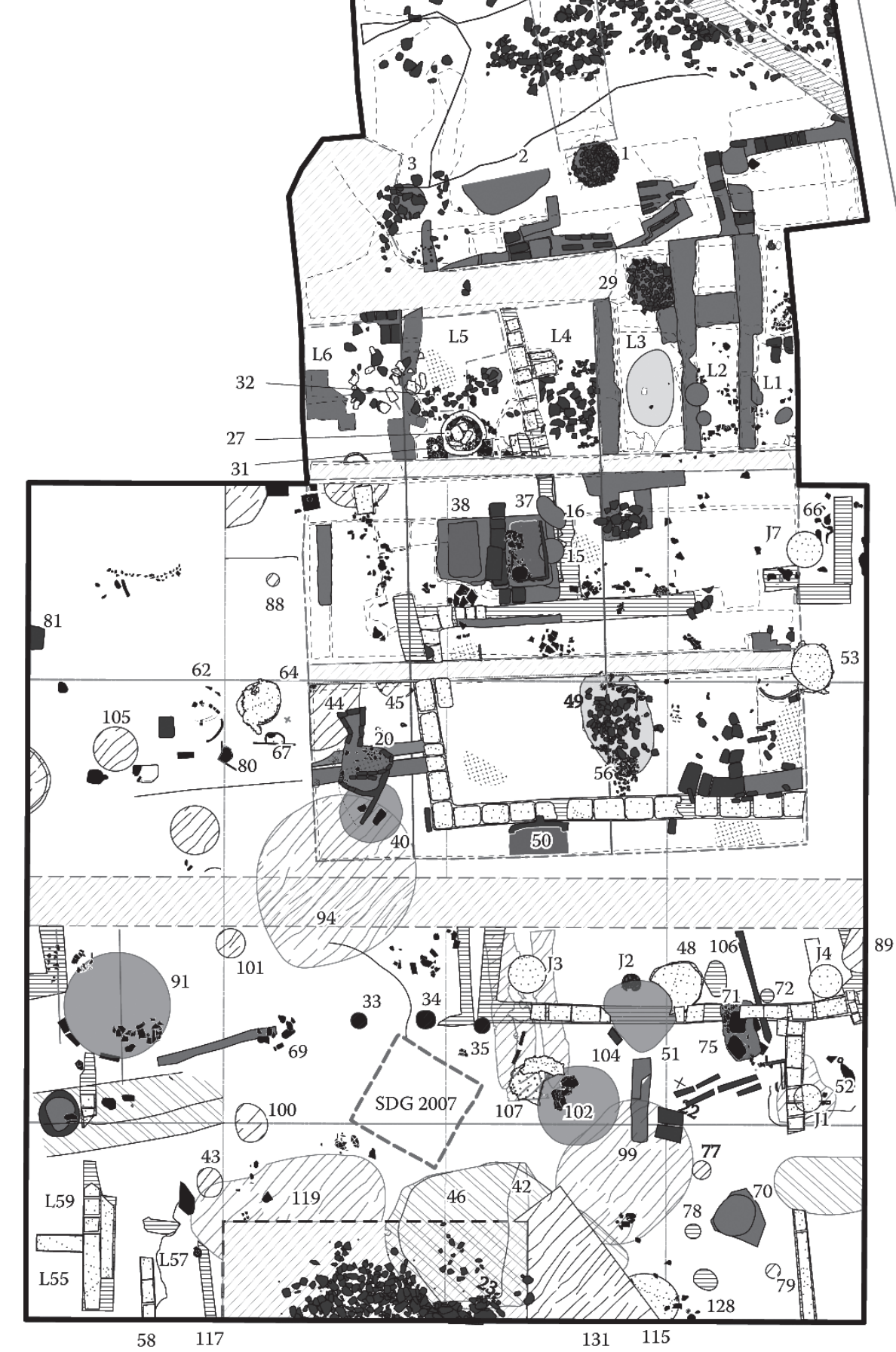


la médecine, la psychologie ou le droit. Dans certains pays européens, il n'existe aucune norme définissant l'archéologue - dans d'autres, une définition légale est en vigueur : avec l'essor de l'archéologie préventive, la tendance est clairement à la définition (et donc au contrôle) des conditions d'accès à la pratique de l'archéologie. Dans la plupart des cas, c'est le diplôme universitaire minimum qui joue un rôle déterminant.

Mis à part les cas du Royaume-Uni et de l'Irlande (qui ont à voir avec le système du common law et la commercialisation de l'activité archéologique) il faut noter que la relative cohérence du tableau [Fig.3] découle de l'harmonisation progressive des structures et des types de cursus universitaires effectuée depuis 1999 et le processus de Bologne : cette uniformisation n'est pas nécessairement synonyme de qualité ou d'égalité de formation à travers l'Europe. Au-delà du diplôme, les archéologues se définissent également par leurs expériences professionnelles. Cette notion d'expérience, associée principalement au terrain (le savoir-faire), est l'une des conditions mentionnées dans la plupart des pays pour l'attribution de permis, laissant l'autorité ad hoc juge de la nature et de la durée de l'expérience requise ou reconnue.

En France, si la reconnaissance des techniciens et des spécialistes est inscrite dans les textes, ils sont tous considérés comme des archéologues. Cette subtilité n'est pas stérile, elle montre au contraire une convergence des savoirs et des savoir-faire au sein de la discipline. La construction française de l'archéologie par le terrain autant que par le concept, associée à une tradition universitaire particulièrement non élitiste, a forgé une profession plus homogène qu'ailleurs. L'archéologue préventif français est le fruit de ce cadre. L'Inrap fait donc figure d'exception socioprofessionnelle dans le panorama de l'archéologie préventive en Europe.

Même s'il n'existe pas (et qu'il n'existera sans doute jamais) de définition unique et sans ambiguïté de l'archéologue professionnel en Europe, il devrait être possible d'arriver à une identification suffisamment ample et pertinente de critères applicables à l'échelle du continent. Ceci permettra de compléter et de renforcer l'approche qualitative de la profession archéologique par une approche quantitative. Une fois le nombre d'archéologues par pays défini de façon comparable - ainsi, techniciens compris, quelque 6800 en Angleterre, 4. 000 en Allemagne et 3500 en France (données 2008) - il devient possible de corréler ces chiffres avec une gamme d'indicateurs démographiques, géographiques, économiques et culturels, à l'échelle européenne : nombre d'archéologues par rapport à la population du pays, sa population active, sa population diplômée ; nombre d'archéologues par surface, par densité d'habitation, par réseau de transport; nombre d'archéologues par produit intérieur brut, par budgets d'aménagement du territoire et de BTP, par budgets dédiés à la culture et à la recherche, etc. À titre d'exemples, les deux encadrés suivants illustrent les premiers résultats des analyses.
Ces résultats quantitatifs pourront être corrélés à d'autres, telles les surfaces étudiées en diagnostic et en fouille, les surfaces « artificialisées » selon des données géo-satellitaires, mais aussi les budgets des opérateurs archéologiques, le nombre d'interventions, de rapports, de publications. Une fois rédigées et diffusées, ces données permettront, aux membres du projet ACE et à tous ceux qui le souhaitent, de mieux explorer les dimensions pratiques, scientifiques et patrimoniales de l'archéologie dans l'Europe contemporaine.

\section{L'archéologie préventive française, support et modèle pour la formation archéologique en Azerbaïdjan : l'exemple du site de Mentesh-Tépé}

\author{
Bertille Lyonnet \\ CNRS, UMR 7192 "Proche-Orient, Iran, Caucase : diversités \\ et continuités ", céramologue \\ Laure Pecqueur \\ Inrap, archéo-anthropologue \\ Pascal Raymond \\ Inrap, topographe \\ Anaïck Samzun \\ Inrap, UMR 7041 « Équipe Protohistoire européenne », archéologue
}

L 'Azerbaïdjan est sorti de la tutelle de l'Union soviétique après avoir acquis son indépendance en 1991. Ce pays est actuellement en plein développement, notamment grâce à l'exploitation des gisements pétrolifères de la mer Caspienne. L'intensité de sa reconstruction économique se conjugue avec sa volonté de préservation des vestiges archéologiques qui pourraient être détruits lors des travaux d'aménagement du territoire. Or, le pays est riche de sites archéologiques dont l'intérêt scientifique est majeur pour l'ensemble de la communauté scientifique. Il est donc nécessaire que son développement soit soutenu par des échanges qui lui apporteront les savoirs utiles à la valorisation de son patrimoine. La fermeture du pays aux évolutions techniques internationales, en particulier ces dernières décennies, n'a pas permis aux archéologues azerbaïdjanais d'acquérir la formation archéométrique et les compétences spécialisées de l'archéologie qui se sont largement développées en Occident à cette époque. Ces insuffisances de moyens techniques et de savoir-faire spécifiques expliquent le manque de rigueur des fouilles et des recherches afférentes conduites jusque-là. Les relevés précis de terrain, les analyses de l'industrie lithique ou osseuse, de la faune et de la flore, ou encore les examens anthropologiques sont encore très peu développés.

Historique de la mission et des recherches archéologiques en Azerbaïdjan. La mission « Boyuk Kesik», financée au départ par le ministère des Affaires étrangères et européennes, a été créée en 2006 à l'initiative de Bertille Lyonnet, en accord avec l'Institut d'archéologie et d'ethnologie de Bakou. Elle vise à mieux comprendre les relations 

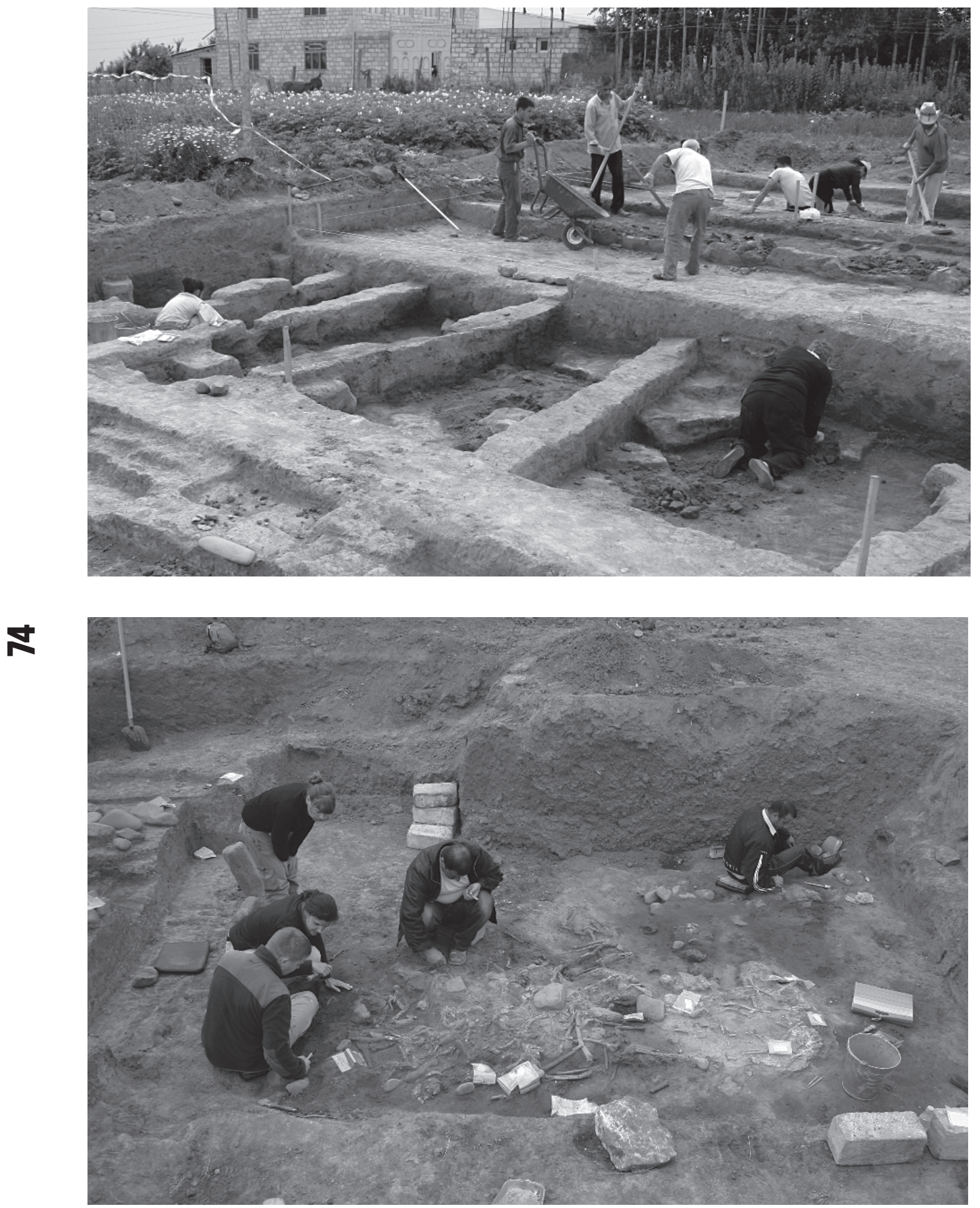

[Fig.3] Vue des architectures chalcolithiques en brique crue en cours de fouille.
[Fig.4] La sépulture collective sous kourgane, en cours de fouille. 
mises en évidence entre les pays du Caucase et la Mésopotamie du Nord au cours du Chalcolithique, en s'intéressant en particulier à la métallurgie (Lyonnet, 2007). Cette mission poursuit les travaux menés précédemment sous sa direction sur la culture de Maikop dans le nord-ouest du Caucase, puis en Géorgie, toujours sur un financement du MAEE.

En 2007, un LiA (Laboratoire international associé) «AzArLi » (Azerbaïdjan, Archéologie et Linguistique) a été créé par un accord entre le CNRS et l'Académie des sciences d'Azerbaïdjan et d'autres partenaires, dont l'Inrap. Il regroupe, côté français, les membres de la mission ainsi que des géologues et des linguistes, et côté azerbaïdjanais, une petite équipe du musée de l'Institut d'archéologie. Il est codirigé par B. Lyonnet et F. Guliyev et vise des échanges de chercheurs à des fins de formation. Depuis 2010, la mission bénéficie aussi du financement de l'ANR pour le programme franco-allemand « Ancient Kura », établi pour une durée de trois ans, auxquels nous sommes également rattachés.

Grâce à un accord préalablement établi par B. Lyonnet entre son équipe CNRS et l'Inrap, il a été possible de faire participer des archéologues azerbaïdjanais, venus dans le cadre des échanges du LiA, à divers travaux de sauvetage en Île-deFrance. Certains ont participé à des opérations de fouille préventive (notamment la fouille de Gonesse dirigée par P. Granchon, Inrap) et se sont familiarisés avec succès au décapage à la pelle mécanique et à la fouille manuelle, d'autres se sont initiés à la DAO-PAO, et le codirecteur du LiA, Farhad Guliyev, s'est particulièrement intéressé à l'organisation et au savoir-faire de l'Inrap. En Azerbaïdjan, sur le terrain, les fouilleurs Inrap ont également encadré des étudiants.

Le financement ANR a permis de lancer, en sus du projet initial sur le Chalcolithique, toute une série de recherches qui permettront de mieux connaître l'environnement entre le $\mathrm{III}^{\mathrm{e}}$ et le $\mathrm{IV}^{\mathrm{e}}$ millénaire et le mode de vie des populations, mais aussi de voir quelles ressources locales furent exploitées alors, tant du point de vue des minerais que des matériaux, comme l'obsidienne. L'ensemble de ces travaux devrait permettre de mieux définir ces cultures, encore mal connues, et de mieux comprendre leurs relations avec la Mésopotamie.

Les recherches sur cette problématique sont conduites par une équipe pluridisciplinaire d'une quinzaine de personnes comprenant, outre une petite équipe Inrap, des spécialistes en géomorphologie, paléobotanique, archéo-zoologie, céramologie, archéo-métallurgie, étude typologique et fonctionnelle des assemblages lithique et de l'industrie osseuse, tracéologie, anthropologie, datations par le carbone 14 et autres méthodes.

Le champ principal de nos investigations, depuis 2007, est le site de Mentesh-Tépé dans le district de Tovuz, dans l'ouest de l'Azerbaïdjan [Fig.1]. Comme la plupart des sites anciens de la région, il n'est pas situé au bord de la Kura, mais à plusieurs kilomètres de là, sur l'une des branches d'un cours d'eau affluent venant du Petit-Caucase.

Le tépé tel qu'il avait été vu dans les années 1960 n'était qu'une petite butte d'une cinquantaine de mètres de diamètre et d'un mètre de haut, mais il avait été depuis entièrement arasé pour faire place à un vignoble, lequel a été arraché à son tour. Seule la présence de tessons en surface et le souvenir de quelques habitants ont permis de le retrouver sous un jardin potager. Un sondage fait en 2007 a vite montré que des structures architecturales et du matériel étaient enfouis sur une hauteur de près de $2 \mathrm{~m}$ sous la surface actuelle.

Les fouilles entreprises à partir de 2008 ont mis au jour un établissement chalcolithique de la seconde moitié $\mathrm{du} \mathrm{v}^{\mathrm{e}}$ millénaire avant notre ère comprenant au moins trois phases architecturales en briques crues (une première, à l'architecture circulaire rappelant celle du Néolithique local, puis deux autres phases, avec des bâtiments rectangulaires), associées à des structures de type fours ou foyers construits et à des silos [Fig.2]. Le mobilier est abondant et comprend beaucoup de céramique, de l'industrie lithique (principalement en obsidienne), mais aussi un certain nombre d'objets en cuivre, ainsi que des artéfacts liés à la métallurgie, depuis le minerai (azurite pilée) jusqu'au moule ou lingot, en passant par les scories et qui attestent, ainsi, l'existence de toutes les étapes de la chaîne opératoire métallurgique sur le site.

Après un long abandon de près de mille ans, le site fut ensuite réutilisé une première fois autour de 3000 avant notre ère pour y enfouir une sépulture collective qui fut recouverte d'un chapeau de galets à la manière des kourganes. Cette chambre et les installations rituelles annexes ont fortement endommagé une partie du site, quasiment jusqu'au sol vierge. Si le nombre d'individus qu'il contenait est très élevé (une cinquantaine), le mobilier funéraire est, lui, relativement pauvre et attribué à la culture kuro-araxe du début de l'âge du Bronze.

Un peu plus tard, au cours de la première moitié du III $^{\mathrm{e}}$ millénaire, d'autres tombes, désormais individuelles, furent creusées ailleurs dans les niveaux chalcolithiques. Outre cette fonction essentiellement funéraire (d'autant qu'un autre kourgane semble apparaître dans une autre partie du site), il est possible qu'il y ait aussi eu un habitat à cette époque, comme pourraient l'attester de vastes fosses contenant des vases presque entiers brisés et de grandes meules. Si aucune des structures architecturales fouillées n'est attribuable avec certitude à cette période Kuro-Araxe, il n'est pas exclu qu'il y en eût dans les niveaux supérieurs arasés du tépé.

Seront évoquées ici plus précisément les interventions et les expériences vécues depuis trois campagnes en partenariat avec les équipes locales composées d'archéologues, d'étudiants et d'ouvriers.

Sensibilisation à l'archéologie de terrain et à la post-fouille. L'archéologie en Azerbaïdjan n'a pas profité de la révolution informatique qui, en quelques années, a totalement transformé le système d'enregistrement et d'exploitation des données partout en Occident. On notera, toutefois, 
que l'archéologie préventive n'y est pas inconnue puisque l'aménagement de pipe-lines pour l'expédition de gaz et de pétrole vers l'ouest a amené des archéologues locaux à développer des fouilles de sauvetage.

À Mentesh-Tépé, notre rôle consiste donc non seulement à fouiller, mais aussi à initier des étudiants et des jeunes archéologues. D'une part, sur le terrain, nous leur montrons nos méthodes, avec relevés, enregistrement des données, compréhension de la stratigraphie et des comblements, etc., sachant que la fouille de la brique crue est complexe [Fig.3]. D'autre part, après la fouille, nous leur apprenons le conditionnement du mobilier, son enregistrement et son analyse. De petits ateliers sont organisés pour permettre à chacun de s'initier à l'informatique, à l'étude de la céramique ou des assemblages lithiques, au dessin, etc.

La plupart des étudiants, voire certains jeunes archéologues, n'ont pas d'expérience de terrain et l'approche de cet environnement ne se fait pas pour eux sans difficultés et parfois du découragement. Souvent déroutés par les conditions climatiques et par le côté « sale » et ingrat des tâches à accomplir, certains refusent ou sont réticents à effectuer des relevés ou à dégager des structures et préfèrent une observation plus ou moins passive. La timidité, la crainte de l'échec ou d'une situation nouvelle expliquent en partie ces réactions pour nous déconcertantes.

La barrière de la langue constitue également un obstacle non négligeable. Aucun de nous ne parle l'azerbaïdjanais, de la famille des langues turques, en sorte que c'est le russe et parfois l'anglais qui sont utilisés, sans être toujours parfaitement maîtrisés d'un côté ou de l'autre, conduisant parfois à des malentendus ou des contresens. À ceci s'ajoutent une formation universitaire principalement livresque et la montée d'un sentiment nationaliste.

Pour toutes ces raisons, nous empruntons des chemins de traverse pour expliquer nos approches et méthodes occidentales en essayant de ne pas heurter l'autre et de ne pas nous trouver nous-même en situation d' " évangélisation », c'est-à-dire à contraindre autrui à accepter aveuglément notre façon de procéder. Au fil des ans, on observe que de plus en plus d'étudiants prennent volontiers part aux activités proposées et font preuve d'enthousiasme et d'initiative. L'anglais est également de plus en plus usité.

Nous espérons pouvoir créer, au cours d'une de nos prochaines campagnes de fouille, un atelier plus particulièrement dévolu au lithique avec démonstration de taille, et analyse typologique, fonctionnelle et tracéologique des outils trouvés, ce qui conduirait les étudiants à mieux comprendre la terminologie utilisée et à savoir les dessiner.

Premiers pas de l'archéo-anthropologie funéraire en Azerbaïdjan. En France, le développement de l'archéo-anthropologie funéraire est assez récent. Il a bénéficié du renouvellement des réflexions méthodologiques qui s'est effectué à l'initiative d'André LeroiGourhan avec notamment la fouille de l'hypogée des Mournouards (Leroi-Gourhan, Bailloud, Brézillon, 1962). C'est ainsi que, à partir des années 1960, le travail de nombreux archéologues, parmi lesquels Claude Masset et Jean Leclerc, a permis de considérer le squelette humain comme objet archéologique à part entière alors qu'auparavant seuls l'architecture de la tombe et les objets accompagnant le mort étaient étudiés. Les vestiges osseux humains, qui sont finalement la raison d'être de la tombe, ont retrouvé dès lors leur place centrale.

À la fin des années 1970, un protocole novateur d'analyse de la mise en place des dépôts a été développé par Henri Duday. Il s'agit d'une approche dynamique des sépultures où sont restitués les gestes funéraires et le processus taphonomique qui crée des distorsions entre l'agencement initial de la tombe et la position du squelette au moment de la fouille. Cette démarche consiste donc à observer l'agencement des os dans la sépulture, ce qui passe par l'identification précise des pièces osseuses présentes (entières ou fragmentées) et le repérage de leur position exacte, de leur orientation anatomique et de leurs relations avec les autres éléments (osseux, mobilier, architecture, etc.). Non seulement la connaissance de l'anatomie humaine est indispensable pour ce travail mais, de plus, face à l'impossibilité de restituer l'ensemble de ces informations a posteriori, il est impératif que tout se fasse au moment de l'intervention sur le terrain.

La découverte fortuite d'une sépulture collective sous kourgane à Mentesh-Tépé a été l'occasion de mettre en œuvre, pour la première fois en Azerbaïdjan, les méthodes de l'archéoanthropologie funéraire telles qu'elles sont appliquées en France. Les délais imposés par la mise à disposition des terrains, accessibles un à deux ans au maximum, font que le dégagement de la chambre funéraire s'est effectué dans l'urgence. L'intervention d'un anthropologue confirmé dans la fouille et l'étude de sépultures complexes a été nécessaire. Toutefois, l'enjeu d'une telle mission ne porte pas seulement sur la fouille de la tombe, mais aussi sur l'initiation aux méthodes d'étude des sépultures (collectives notamment) en présentant les techniques de fouille et tous les résultats qu'elles permettent d'obtenir, tant sur le monument que sur les défunts.

\section{État des lieux de l’archéologie funéraire} en Azerbaïdjan. En Azerbaïdjan, les fouilles des sépultures privilégient l'architecture et le matériel funéraire au détriment des restes humains. Le dégagement des os se limite au seul niveau supérieur de la couche d'inhumation, pour les sépultures collectives du moins, et, au mieux, seul un croquis général du squelette est établi. Les crânes sont comptabilisés pour estimer le nombre d'individus inhumés et sont éventuellement les seuls éléments à être analysés, selon la vieille méthode anthropologique soviétique. Le reste de la sépulture est vidé de manière expéditive et les ossements sont en général immédiatement réenfouis à proximité du site, le but étant, avant 
tout, de prélever les objets qui accompagnent les défunts. En effet, ces sépultures sont connues pour contenir souvent un mobilier abondant et riche.

Les échantillons archéologiques issus des sépultures se limitent donc souvent seulement à des crânes, sortis de leur contexte, à partir desquels on cherche à définir les caractéristiques morphologiques des individus concernés ou, pour parler plus crûment, les « races humaines ». En aucun cas la fouille n'a pour objectif la compréhension des dépôts.

Il est donc aujourd'hui primordial de montrer l'importance d'une véritable étude des vestiges humains qui passe, de prime abord, par la formation, deuxième volet de notre intervention en Azerbaïdjan.

Formation à l'étude des sépultures : obstacles et espoirs. Au-delà des problèmes méthodologiques, la fouille et l'étude des sépultures se heurtent à un tabou : de nombreuses personnes n'aiment pas manipuler les ossements humains, voire ne veulent pas les toucher du tout. Si cette répulsion ne représente pas un handicap en France, l'archéologie funéraire attirant, au contraire, de plus en plus de monde, il en va différemment en Azerbaïdjan. Pour quelques-uns, la manipulation des os humains est acceptée, même si elle est suivie d'un lavage méthodique des mains à l'alcool à $90^{\circ}$. Pour la plupart, néanmoins, l'idée même de toucher des os humains révulse et il est alors très difficile de trouver des candidats, archéologues ou étudiants, qui s'intéressent aux pratiques funéraires et qui acceptent de se former. À ce tabou s'ajoute la formation universitaire qui dispense uniquement un enseignement d'anthropologie physique dans le but de caractériser les groupes humains.

Pourtant, l'étude des sépultures n'a pas cette vocation. Les vestiges humains, qui sont les seuls éléments en relation directe avec la biologie et la démographie, renseignent, d'une part, sur la mort car, en l'absence de sources écrites, ce sont souvent les uniques témoins de l'idéologie funéraire de l'époque concernée, et, d'autre part, sur les morts (qui ? comment? où ?) et, à travers eux, sur la société des vivants et sur sa structure socioéconomique. Un travail est donc nécessaire pour sensibiliser les archéologues azerbaïdjanais à l'existence de véritables méthodes d'étude des sépultures, ce qui doit se faire au tout début de la formation universitaire.

Au cours des différentes campagnes qui ont eu lieu à Mentesh-Tépé, nous avons initié nos collègues archéologues et les étudiants aux techniques de fouille de sépultures sur le terrain [Fig.4]. Parallèlement, l'enregistrement des informations dans la base de données et le plan général de la couche d'inhumation ont été présentés lors de petits ateliers. Un premier contact avec les ossements s'est même effectué à travers leur lavage et leur conditionnement, mais aussi par la participation d'étudiants à une activité de remontage de crânes. Au-delà de ces initiations, c'est une véritable formation qui serait nécessaire pour que les futurs archéo-anthropologues azerbaïdjanais deviennent autonomes. La mise au jour en 2010 de ce qui semble être une nouvelle tombe collective sous kourgane à Mentesh-Tépé sera l'occasion de mettre en place, dès septembre 2011, une initiation en archéo-anthropologie funéraire. Le but sera de montrer l'ensemble de la chaîne opératoire, du terrain à l'étude. Cela passera par l'apprentissage de la fouille et du démontage des différents niveaux d'inhumation, mais aussi par l'enregistrement des informations (photographies, fiches de conservation, notes, prises d'altitude, etc.). Sur le terrain, nous envisageons de constituer de petites équipes comprenant un archéoanthropologue français encadrant un ou deux étudiants. Cela sera complété par une formation en laboratoire, l'examen des sépultures passant avant tout par la reconnaissance des ossements.

L'étude de la sépulture collective sous kourgane fouillée en 2008 et 2009 n'est pas encore achevée. Les résultats présentés aux Azerbaïdjanais devraient les convaincre de l'importance et de la nécessité de fouiller avec de telles méthodes au vu des informations qu'elles apportent sur les morts, et donc indirectement sur les vivants. Notre participation aux fouilles du site de Mentesh-Tépé s'avère être, finalement, d'autant plus enrichissante que cette mission a aussi une vocation de sensibilisation et de formation des équipes locales aux méthodes de l'archéo-anthropologie funéraire.

Sensibilisation à la topographie et au dessin lithique. Ces dernières années, l'évolution des moyens techniques de relevés a connu de grandes transformations. La topographie est utilisée comme une discipline pivot qui apporte la base structurante permettant le redressement de photographies, la modélisation $3 \mathrm{D}$ ou la gestion de données sur SIG. Sur les chantiers d'archéologie préventive, ces techniques donnent lieu à des économies de temps de travail et apportent une vision nouvelle des vestiges. Nous avons ainsi eu l'occasion d'appliquer la modélisation de terrain pour restituer en $3 \mathrm{D}$ des vestiges dans leur environnement stratigraphique. Ces méthodes, en offrant une vision exacte des faits, permettent d'affiner une hypothèse ou parfois livrent des informations sur l'organisation spatiale qui n'a pas été perçue sur le terrain. La puissance de calcul des ordinateurs et des logiciels spécifiques nous aide à produire des documents toujours plus précis.

Une des ambitions de la mission de MenteshTépé était de démontrer l'intérêt et la place de la topographie telle qu'elle est utilisée en archéologie préventive. La topographie a ainsi servi de cadre à tous les relevés. Nous avons tenté d'exploiter un maximum de solutions techniques disponibles en lien avec la topographie. La prise de points a tout d'abord été utilisée de façon traditionnelle pour le calage des minutes de terrain. Nous avons aussi pu montrer qu'avec un enregistrement tridimensionnel des faits, il était plus aisé de démonter rapidement les vestiges et par conséquent d'accélérer le rythme de la fouille. Par ailleurs, au vu du caractère exceptionnel de certaines structures auxquelles nous étions confrontés, nous avons pu rapidement exploiter la topographie comme base 
pour le redressement de photographies et la modélisation de terrain.

Cette fouille se déroulant sur plusieurs années, c'est en partie de la qualité des enregistrements que dépendra l'interprétation finale des vestiges. Notre temps de fouille sur le site étant limité à seulement trois semaines par mission, le rythme des relevés dans ce contexte est plus soutenu que sur de nombreux chantiers d'archéologie préventive. Ce fut notamment le cas pour la sépulture collective sous kourgane qu'il a fallu fouiller en cinq semaines sur deux ans. La fouille d'une telle structure en France aurait probablement duré trois mois avec cinq anthropologues.

Les relevés ne devant pas entraver l'avancement de la fouille, la méthode la plus efficace a consisté à utiliser le redressement de photographies calées sur une topographie assez fine. Ce mode de relevé est ensuite appliqué à la majorité des vestiges comme les amas de galets, les plates-formes de tessons ou les foyers aménagés. La multiplication de ce genre d'exercices nous a permis d'affiner cette méthode. Ainsi, désormais, nous maîtrisons mieux la prise de clichés, le positionnement des points de calage et la gestion de l'ensemble des informations. Ce travail nous a aussi permis de cerner les limites de l'exercice au vu des moyens dont nous disposions.

Nos collègues et étudiants azerbaïdjanais ont pu apprendre les bases du relevé manuel de structures et le comparer avec le résultat obtenu par des méthodes plus innovantes. Ils se sont ainsi initiés à l'utilisation du théodolite et ont participé à plusieurs ateliers autour de la chaîne graphique. Nous avons abordé à cette occasion l'utilisation et les spécificités de logiciel comme Auto CAD, Adobe Illustrator ou Arc Gis. Ces exercices leur ont permis de comparer différentes approches pratiques pour l'enregistrement et la restitution de l'information spatiale. Il reste que, pour avancer davantage, il serait nécessaire qu'ils aient à l'université une formation de base sur tous ces logiciels, ce qui n'est pas encore le cas.

Par ailleurs, la transposition de ces méthodes sur des fouilles à l'étranger permet de les tester dans un contexte particulier et de faire évoluer leur utilisation. Les contraintes particulières des fouilles en Azerbaïdjan tiennent à l'autonomie totale de la mission. Loin de tout, il n'est pas possible de contacter un spécialiste, réparer du matériel ou chercher une pièce chez un collègue. Ces conditions nous permettent de valider la simplicité et l'efficacité d'une méthode. En effet, pour être performantes dans ces conditions, ces pratiques ne doivent nécessiter qu'un minimum de ressources tout en permettant un enregistrement de qualité des informations. En plus de ces contraintes matérielles, la nature des structures nous impose un rythme de fouille et un démontage rapide des vestiges. Nous n'avons que trop rarement l'occasion d'intervenir sur le type de structures que nous fouillons sur le site de Mentesh-Tépé. Tout ce que nous pouvons observer est dans un état de conservation que nous ne connaissons pas en France. Le cœur du site est stratifié sur deux mètres et les bâtiments en brique crue sont conservés sur plusieurs assises. Le mobilier est d'une variété, d'une abondance et d'une qualité que nous ne connaissons pas en France pour ces périodes.

Notre mission contribue également à la formation d'étudiants en archéologie de l'Académie des sciences de baku. C'est ainsi que nous leur avons montré les techniques de dessin de mobilier lithique qui font référence à une codification descriptive qu'ils ne maîtrisent pas. Or, nous disposons d'une grande diversité de pièces en obsidienne et en silex. Ce matériel nous a permis d'aborder avec eux les différentes techniques de débitage, les fonctions particulières de certains outils, et d'identifier des stigmates visibles de leur utilisation. Au travers du dessin lithique et de ses principes de représentation, nous leur avons apporté des clés permettant l'interprétation de ce type de mobilier. Ainsi, nous avons pu les sensibiliser à des pièces auxquelles ils ne prêtaient pas habituellement attention.

Le bénéfice que nous retirons de notre expérience en Azerbaïdjan est important pour nos pratiques quotidiennes. Ce travail nous familiarise avec des matériaux ou des structures que nous rencontrons rarement, nous apporte un regard différent sur certains faits et la façon de les aborder. Ces missions, par leur intensité, permettent de valider des méthodes et nous montrent les limites de certains exercices.

Perspectives. L'archéologie à l'étranger et l'archéologie préventive sur le territoire national présentent des similitudes et sont étroitement liées. En effet, l'analyse et la compréhension des sites doivent, dans les deux cas, se réaliser dans un laps de temps limité, nous contraignant à faire rapidement des choix dans la stratégie de fouille. De plus, comme en témoigne le choix de nombreux responsables de fouilles à l'étranger, ce sont les qualités dans les méthodes, techniques et savoir-faire de l'Inrap qui sont recherchés. Grâce à eux, nous aidons les archéologues locaux à acquérir une compétence et une maîtrise dans les derniers développements des techniques de fouille et d'analyses du matériel. Mais, en retour, nous gagnons aussi en renommée internationale, le nom de l'Inrap étant diffusé dans la presse et les médias locaux. Enfin, notre démarche s'inscrit dans une volonté de sensibiliser les équipes locales à la fouille de sauvetage car, chaque année, de nombreux sites sont découverts au gré des aménagements, ou détruits sans laisser de traces. Aussi est-il important de procurer aux acteurs de l'archéologie locale les moyens de sauvegarder une partie de leur patrimoine par la transmission des méthodes de fouille et d'étude. Nous sommes conscients que notre travail n'est qu'une petite pierre à l'édifice de l'archéologie du pays, mais, comme S. Cleuziou le précisait si bien, « le passé est celui de tous et n'est pas limité à des frontières » (Cleuziou, 1985). 
Cleuziou S., 1985, « L'archéologie française à l'étranger : redéfinition ou déclin? » Les Nouvelles de l'Archéologie, ${ }^{\circ}$ 20, p. 8-25.

DUDAY H., 1995, « Anthropologie "de terrain", archéologie de la mort », in La Mort, passé, présent, conditionnel, colloque du Groupe vendéen d'études préhistoriques, La Roche-sur-Yon, 1994, GVEP, p. 33-58.

Leroi-Gourhan A., Bailloud G., Brezillon M., 1962, « L'hypogée I des Mournouards (Mesnil-sur-Oger, Marne) », Gallia Préhistoire, t. 5-1, p. 23-123.

LYONNET B., 2007, « La culture de Maikop, la Transcaucasie, l'Anatolie orientale et le Proche-Orient : relations et chronologie », in Lyonnet B. (DIR.), Les Cultures du Caucase (VI-III millénaire avant notre ère). Leurs relations avec le Proche-Orient, Paris, CNRs éditions, p. 133-161.

\section{Une coopération franco-chinoise: les fouilles du site de Gongying}

\author{
Alain Thote \\ École pratique des hautes études (4 ${ }^{e}$ section), chef de la mission \\ archéologique "Royaume de Chu », UMR 8155 " Centre \\ de recherches sur les civilisations de l'Asie orientale» \\ Karine Michel \\ Inrap, Grand-Est/Nord, Metz, céramologue
}

$\mathrm{H}$ istoire de l'archéologie chinoise. On connaît encore assez mal l'archéologie chinoise en France, en dépit de quelques expositions et d'articles qui se focalisent sur les sites les plus remarquables. En fait, c'est bien souvent l'aspect spectaculaire des découvertes que retient le public, tel qu'il est médiatisé par les journalistes qui en rendent compte. Pour comprendre ce qui se passe aujourd'hui en Chine dans ce domaine, une petite révolution, il convient de rappeler brièvement quelques faits. Très tôt, on s'y est intéressé à l'Antiquité, non pas avec la seule intention de collectionner de beaux objets, mais pour comprendre une société qui appartenait à une époque vue comme un âge d'or, et s'en inspirer. On a d'abord collationné les textes ; on les a publiés sous forme manuscrite, puis imprimés ; on les a annotés, commentés du début de l'Empire (221 avant notre ère) jusqu'à nos jours. Parallèlement, mais dans une bien moindre mesure, on s'est intéressé, dès le $\mathrm{XI}^{\mathrm{e}}$ siècle, aux vestiges matériels pour les étudier d'une façon scientifique. C'est à cette époque que parurent les premiers catalogues imprimés d'antiquités réunissant les plus belles pièces archéologiques connues. Certains de ces catalogues sont parvenus jusqu'à nous : chaque objet y est présenté par un dessin au trait et est accompagné d'une légende donnant ses dimensions, une description sommaire, son lieu de découverte, le nom de son propriétaire. On y reproduit encore son inscription, quand il en a une, sous deux formes : un estampage et une transcription en caractères standards, puisque les graphies avaient changé entre-temps.

Les missions scientifiques menées par les Occidentaux et les Japonais sur le sol chinois à partir de la fin du XIX ${ }^{\mathrm{e}}$ siècle, le pillage de ses trésors par des antiquaires peu scrupuleux alimentant le marché national et international durant la première moitié $\mathrm{du} \mathrm{xx}^{\mathrm{e}}$ siècle, ont eu deux effets importants sur l'archéologie de ce pays. D'une part, les Chinois ont entrepris, à leur tour, des fouilles à partir de 1928. Pour ce faire, ils ont choisi le site d'Anyang, la dernière capitale des Shang (environ $\mathrm{XVI}^{\mathrm{e}}$ siècle-1050 avant notre ère) et utilisé les méthodes qu'ils avaient apprises des Occidentaux. D'autre part, ils ont tenu à les entreprendre indépendamment de ces derniers. De 1949 jusqu'au début des années 1980, la Chine a développé son archéologie en vase clos, sans tenir compte des avancées faites ailleurs dans ce domaine. Les instructions données aux archéologues ont encadré et encadrent toujours, d'une manière rigide, leur travail. Elles sont uniformément appliquées sur tout le territoire : fouilles en carrés de $5 \times 5 \mathrm{~m}$, ou de $10 \times 10 \mathrm{~m}$, avec une berme d'un mètre de large mordant sur deux côtés, au nord et à l'est, chantiers strictement orientés au nord, etc. Ce sont, sous une forme plus systématique, les méthodes élaborées par sir Mortimer Wheeler (1890-1977) et ses prédécesseurs. D'autre part, pendant toutes ces années - et même encore aujourd'hui les recherches sont restées très dépendantes de la lecture que l'on faisait des sources textuelles anciennes, sur lesquelles se sont penchées des générations de lettrés. On tend d'abord à chercher dans les vestiges la confirmation de ce que l'on connaît par les textes classiques, les annales et les chroniques, sans toujours prendre en compte que ces sources ne sont pas nécessairement dignes de foi. L'archéologie a longtemps servi à illustrer ce que l'on connaît de l'histoire chinoise. Aujourd'hui c'est beaucoup moins le cas.

\section{L'archéologie chinoise aujourd'hui.}

L'ouverture économique de la Chine, à partir de 1978-79, a eu pour effet de modifier les conditions dans lesquelles sont opérées les fouilles. Les grands chantiers de travaux publics conduisent à faire d'innombrables découvertes, déclarées ou passées sous silence. Les échanges avec l'étranger ont permis aux archéologues chinois de mesurer le retard scientifique pris lorsque le pays était fermé à tout apport extérieur. Les moyens humains et financiers ont été améliorés dans le but de faire connaître la civilisation chinoise dans ses aspects les plus prestigieux. En particulier, on s'emploie aujourd'hui, à montrer que cette civilisation a un âge vénérable de cinq mille ans, à l'égal des plus grandes civilisations connues de par le monde.

On ne peut ici dresser un état des lieux: il resterait fort incomplet tant les changements récents sont importants, tout en restant inégaux selon les régions et les moyens mis en œuvre. De plus, la Chine a énormément investi dans la formation de jeunes archéologues, qui souvent la parachèvent en étudiant plusieurs années à l'étranger et surtout aux États-Unis. Elle place aussi des moyens considérables dans la recherche. La diffusion des connaissances est à la fois rapide et riche. Les publications chinoises d'aujourd'hui n'ont rien à nous envier : revues scientifiques à l'échelon national, provincial ou local, rapports complets de fouilles sous la forme de livres illustrés d'excellente qualité. En Chine aussi, on est passé d'une archéologie entièrement financée par l'État, 